\title{
A genome-wide expression profile analysis reveals active genes and pathways coping with phosphate starvation in soybean
}

\author{
Qing Wang ${ }^{1+}$, Jiao Wang ${ }^{1 \dagger}$, Yuming Yang ${ }^{1}$, Wenkai Du', Dan Zhang ${ }^{2}$, Deyue Yu and Hao Cheng ${ }^{1 *}$
}

\begin{abstract}
Background: Phosphorus is one of the most important macronutrients that is required for plant growth and development. However, stress under low-P conditions has become a limiting factor that affects crop yields and qualities. Plants have developed strategies to cope with this, while few genes associated with low-P tolerance have been identified in soybean.

Results: Genome-wide analyses were performed on the roots and leaves of a low-P-tolerant accession and a low-Psensitive accession which were identified by hydroponic experiments under different $P$ treatments. Through comparative analyses on the differently expressed genes, we explored 42 common genes that were highly correlated to low-P stress. The functional classification of these genes revealed 24 Gene Ontology (GO) terms of biological process including response to oxidation reduction, hormone stimuli, and biotic and abiotic stimuli. Additionally, three common pathways were identified.

Conclusions: These results could not only promote the work on the molecular regulation mechanism under low-P stress in soybean, but also facilitate the cultivation of high-phosphorus-acquisition and high-phosphorus-utilization soybean varieties.
\end{abstract}

Keywords: Soybean, Root system, Low-P stress, Phosphorus deficiency, Expression profile

\section{Background}

As an essential constituent of many important compounds that are required for the development and growth of plants, phosphorus $(\mathrm{P})$ actively participates in photosynthesis, respiration, carbohydrate metabolism, energy transduction and other processes [1]. In many agricultural systems, however, the concentration of absorbable phosphorus in soil is insufficient to ensure plant productivity [2].

As one of the most important grain and industrial crops, soybean (Glycine $\max$ L. Merrill) is a vital source of protein for human and animal food. However, soybean growth and yield are limited by varieties of edaphic factors, especially low phosphorus availability in soils [3].

\footnotetext{
* Correspondence: jenny21star@njau.edu.cn

${ }^{\dagger}$ Equal contributors

${ }^{1}$ National Center for Soybean Improvement, National Key Laboratory of Crop Genetics and Germplasm Enhancement, Nanjing Agricultural University, Nanjing, China

Full list of author information is available at the end of the article
}

Under low-P stress, soybean plants become dwarfed, leaf area is reduced, and necrotic spots might appear on lower leaves [4]. Also, flowering time is increased and the number of pods could decrease during the fruiting period [5]. Due to these P-mediated phenotypic effects on soybeans, phosphate fertilizers are used to overcome undesirable potential yield outcomes. Large quantities of phosphate fertilizer may cause environmental pollution as it is easily immobilized in the soil to form chelates or precipitates [6]. In order to alleviate the effects of phosphorus deficiency on soybean growth, it is particularly important for us to search for P-efficient soybean materials and investigate high-phosphorus-efficient genes.

Recently, four major groups of genes induced by low-P stress were reported [7]. One group was involved in sensing low-P signals, including local phosphate sensing that was proposed to occur in the primary root in Arabidopsis thaliana [8], and long-distance phosphate signaling which depends on the activity of transcription factors, such as PHR1, PHL1 [9]. Members of another 
group mediated phosphate distribution by involving an internal regulation of phosphorus homeostasis, including SPX family and PHO1 that contains SPX domain in Arabidopsis [10]. The remaining two groups of genes mainly focus on the acquisition and transportation of phosphate. For example, GmACP1 encodes an acid phosphatase, the overexpression of which in roots could increase the absorption of phosphate in the nutrient solution [11]. The PHT1 family consisting of 15 PHT1 genes in soybean encoded phosphate transporters was demonstrated to be involved in not only directing phosphate uptake in roots, but also transporting phosphorus as phosphate [12, 13]. Generally, the acquisition and transportation of phosphate greatly depends on the root system as root morphology changes under P-deficient conditions to sustain plant growth. This includes an increase in number and length of lateral roots in maize (Zea mays L.) [14], longer and greater number of root hairs in low-P tolerant accessions of barley (Hordeum vulgare L.) and Arabidopsis [15, 16], and relatively longer primary roots in rice (Oryza sativa L.) when a lowphosphorus-related transcription factor OsMYB2P-1 is over expressed [17]. Meanwhile, QTL (quantitative trait loci) mapping associated with soybean phosphorus efficiency have made processes. QTLs related to seed phosphorus content [18], phosphorus efficiency relevant root traits [19], and soybean tolerance to low phosphorus stress based on flower and pod abscission rate [20] have been identified. Furthermore, screening of soybean Pefficient materials identified P-efficient genotypes, such as HP119, HP134, Huaxai 1, Huaxia 2 etc. [21, 22].

As an abiotic stress, low-P conditions may induce a series of complex responses. It was demonstrated that phytohormone signaling pathways responded strongly to phosphate deprivation [23, 24]. The expression of a large set of genes associated with plant hormone signaling either induced or repressed root growth in Arabidopsis and Zea mays when suffering low-P conditions [23, 24]. In addition, changes of the secondary metabolites to low-P stress were shown with an accumulation of anthocyanin under long-term phosphorus deficiency [23]. Similar results were observed in Zea mays with genes related to the phenylpropanoid pathway identified as upregulated or down-regulated under P starvation [24].

A large set of genes and signaling pathways that were associated with low-P stress had been uncovered using the technique of gene expression profiles and transcriptome analyses in Arabidopsis, rice, and Zea mays [23-27]. Recently, Zeng et al. identified phosphate-deficiencyresponsive genes in soybean (Glycine max var. Williams 82) roots by high-throughput sequencing [28]. Selection of representative materials and consideration of different tissues will further reveal genes and pathways associated with low-P stress. Here, we selected a low-P-tolerant accession Chundou (CD) and a low-P-sensitive accession Yunhefengwodou (YH) from 219 soybean accessions through hydroponic experiments. Then microarray chips were performed on the roots and leaves of these two accessions. By analyzing the gene expression data, we identified 42 candidate genes and three common pathways that were induced by low-P stress. Our study provides candidate loci for functional identification of high-phosphorusefficient genes which may be of great significance for cultivating P-efficient soybean accessions.

\section{Results \\ Identification of low-P-tolerant accession CD and low-P- sensitive accession $\mathrm{YH}$}

With hydroponic experiments lasting for 10 days under different $\mathrm{P}$ treated conditions of 219 soybean accessions [29], we found that the soybean accession $\mathrm{CD}$ and $\mathrm{YH}$ showed similar root morphology under $+\mathrm{P}$ treated condition (Hoagland with $1.0 \mathrm{mmol} / \mathrm{L} \mathrm{P}$ ), while showing obvious morphology differences under -P treated condition (Hoagland with $0.01 \mathrm{mmol} / \mathrm{L} \mathrm{P}$ ). We then identified accession $\mathrm{CD}$ as a low-P-tolerant accession, and accession $\mathrm{YH}$ as a low-P-sensitive accession.

Then, we carried out a gradient experiment using full, half and quarter Hoagland's solution and found that the $+\mathrm{P}$ and $-\mathrm{P}$ treated soybean plants showed most obvious morphology difference when grown in half Hoagland. Hence half Hoagland with $0.5 \mathrm{mmol} / \mathrm{L}$ $\mathrm{P}$ and $0.005 \mathrm{mmol} / \mathrm{L} \mathrm{P}$ were considered as normal and low $\mathrm{P}$ treated conditions, respectively. The phenotypic traits of $\mathrm{CD}$ and $\mathrm{YH}$ are shown in Additional file 1: Figure S1. Primary root length was longer in CD than that in $\mathrm{YH}$. The number of root hairs and lateral roots of $\mathrm{CD}$ were much more than those in $\mathrm{YH}$. Microarray experiments were performed on the roots and leaves of $\mathrm{CD}$ and $\mathrm{YH}$.

\section{Significantly differentially expressed genes in different $P$ treated conditions}

Under low P condition, 257 and 11 up-regulated differently expressed genes (DEGs) were found in the roots and leaves of low-P-tolerant accession $\mathrm{CD}$ respectively; while no up-regulated DEGs were found in the roots and leaves of low-P-sensitive accession $\mathrm{YH}$ compared with low-P treatment. For down-regulated DEGs, only 41 DEGs could be found in the roots of CD, 3 DEGs in the roots, and 7 DEGs in the leaves of YH (Table 1).

Generally, the number of DEGs in CD was more than that in $\mathrm{YH}$, not only in the roots but also in the leaves (Table 1), which suggested that $\mathrm{CD}$ could regulate a series of genes to cope with the low-P stress. We found only one common DEG, Glyma20g33710.2, in both the roots of $\mathrm{CD}$ and $\mathrm{YH}$, with the fold change 0.44 and 0.32 
Table 1 The number of DEGs in different $\mathrm{P}$ treated conditions

\begin{tabular}{llllll}
\hline Materials & Tissues & $\begin{array}{l}\text { Number of up- } \\
\text { regulated genes }\end{array}$ & $\begin{array}{l}\text { Number of down- } \\
\text { regulated genes }\end{array}$ & $\begin{array}{l}\text { Up-regulated } \\
\text { fold change }\end{array}$ & $\begin{array}{l}\text { Down-regulated } \\
\text { fold change }\end{array}$ \\
\hline $\mathrm{CD}$ & Roots & 257 & 41 & $2.00-33.78$ & $0.11-0.49$ \\
& Leaves & 11 & 0 & $2.32-5.29$ & - \\
$\mathrm{YH}$ & Roots & 0 & 3 & - & $0.30-0.44$ \\
& Leaves & 0 & 7 & - & $0.038-0.34$ \\
\hline
\end{tabular}

down-regulated in low-P-tolerant accession CD and low$\mathrm{P}$-sensitive accession $\mathrm{YH}$, respectively.

The number of DEGs in the roots was more than that in the leaves. Interestingly, one common DEG, Glyma01g04350.1 named as GmMMP2, which encodes a matrix metalloproteinase, was found both in the roots and leaves of CD. The fold change is 4.10 and 3.59, respectively. Previous studies demonstrated that it was associated with pathogenic infections in soybean [30].

A total of 317 non-redundant DEGs were found between treated and control conditions in the roots and leaves of both soybean accessions used in this experiment. The hierarchical clustering analysis classified the tolerant and sensitive soybean accessions into two clusters; samples cluster and genes cluster. The compactness of the clusters of the three replicates confirmed the high reliability of our experimental design (Fig. 1).

To evaluate the potential functions of the DEGs between low-P stress and normal conditions in both soybean accessions, Gene Ontology (GO) categories were applied for low-phosphorus-induced genes. The three GO terms were categorized as biological processes, molecular functions, and cellular components. We found that DEGs were significantly associated with response to wounding, response to jasmonic acid stimulus, oxidoreductase activity, and cell wall, whether they were upregulated genes or down-regulated genes. Furthermore, we identified pathways that were associated with low-P induced genes with the use of KEGG (Kyoto Encyclopedia of Genes and Genomes). Overall, 45 pathways with $P$ values less than 0.05 were motivated under low-P stress (Additional file 2: Table S1), and most pathways were related to metabolic process. Notably, the most significant 3 pathways that were enriched included phenylpropanoid biosynthesis, methane metabolism and phenylalanine metabolism, all related to the metabolism of secondary metabolites.

\section{Significantly differentially expressed genes between different soybean accessions}

Accessions $\mathrm{CD}$ and $\mathrm{YH}$ showed strong differences in the ability to suffer low-P stress (Additional file 1: Figure S1). There were much more DEGs in CD (309) than in YH (10) (Table 1, Additional file 3: Table S2). To further reveal the DEGs related with low-P condition between $\mathrm{CD}$ and $\mathrm{YH}$, we compared the DEGs between soybean accessions $\mathrm{CD}$ and $\mathrm{YH}$ (Table 2).

DEGs induced by low-P conditions were classified as three categories according to their expression patterns in normal conditions. For 1777 up-regulated genes in roots between $\mathrm{CD}$ and $\mathrm{YH}$ in low-P stress (Additional file 4: Figure S2), the three categories were as follows: 1 ) 941 genes were up-regulated in $\mathrm{CD} / \mathrm{YH}$ in normal conditions, which is consistent with the expression pattern in low-P stress; 2) 10 genes were down-regulated in normal conditions, which showed the opposite expression patterns between normal and low-P conditions; and 3) 826 genes showed no expression difference in normal condition, with the expression difference less than two fold between $\mathrm{CD}$ and $\mathrm{YH}$. By the criterion that genes with two fold expression change between experimental group and control group were identified as DEGs, the expression level in $C D$ was more than two fold higher than that in $\mathrm{YH}$ for the 1777 up-regulated genes (Additional file 4: Figure S2). Among the 1777 upregulated genes, there were 826 genes with no expression difference in normal conditions, the expression difference of which between $\mathrm{CD}$ and $\mathrm{YH}$ was less than two fold. To make the expression difference of $\mathrm{CD} / \mathrm{YH}$ between low-P stress and normal conditions higher than two fold, we chose those genes with larger than 4-fold expression difference between $C D$ and $\mathrm{YH}$ under low-P conditions as candidates. By this criterion, 93 genes with larger than 4-fold expression difference between $\mathrm{CD}$ and $\mathrm{YH}$ under low-P condition from the 826 genes were chosen as candidates. Hence, we got 40, 14, and 38 DEGs from 975, 324, and 526 DEGs, respectively (Table 2). Combined with the genes with opposite expression patterns, they were considered as marked DEGs between CD and YH. Therefore, 196 marked DEGs were obtained. Among these genes, one gene was up-regulated more than 4 fold between $\mathrm{CD} / \mathrm{YH}$ under low-P conditions in both roots and leaves. Thus, there were 195 nonredundant marked DEGs detected in total. Among these marked DEGs, the majority (143 genes, $73.33 \%$ ) were upregulated in $\mathrm{CD} / \mathrm{YH}$ under low-P stress. Additionally, there were 117 and 79 genes expressed differently between $\mathrm{CD}$ and $\mathrm{YH}$ in roots and leaves, respectively. This result suggested more genes were activated in roots than in leaves under low-P stress during seedling stage. 


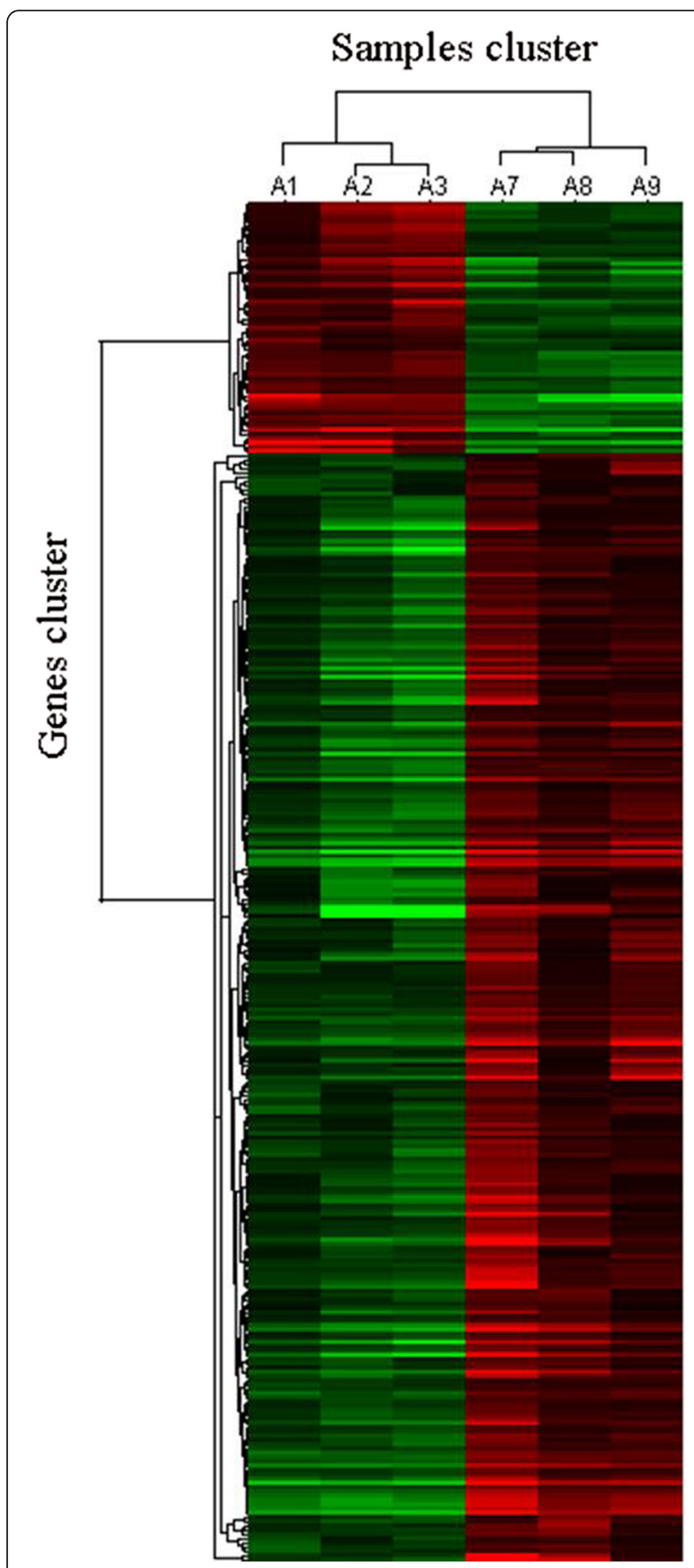

Fig. 1 Hierarchical clustering of 319 differentially expressed genes in different $\mathrm{P}$-treated conditions. $\mathrm{A} 1, \mathrm{~A} 2$ and $\mathrm{A} 3$, three biological replicates of control groups ( $0.5 \mathrm{mmol} / \mathrm{L} \mathrm{P}) ; \mathrm{A} 7, \mathrm{~A} 8$ and $\mathrm{A}$ 9, three biological replicates of treatment groups $(0.005 \mathrm{mmol} / \mathrm{L} \mathrm{P})$. Red and green indicate high and low expression levels, respectively

We performed the enrichment of GO and KEGG analysis for these 195 marked DEGs. GO analysis showed that these genes were mainly involved in secondary metabolic processes, for example flavonoid biosynthesis. They were also involved in response to hormone stimuli (jasmonic acid, auxin, ethylene, and salicylic acid), and defense and abiotic stimuli (oxidative stress, salt stress response, and hypersensitive response). In addition, they were involved in electron carrier activity and peroxidase. KEGG analysis revealed the pathways enriched were mostly associated with the metabolism of secondary metabolites, including phenylpropanoid biosynthesis, methane metabolism, phenylalanine metabolism, flavonoid biosynthesis and so on (Additional file 2: Table S1).

The 317 DEGs in different P-treated conditions were considered as genes in response to low-P stress. The 195 marked DEGs in different soybean accessions were not only induced by low-P stress but also by materialsvariation dependent upon the response to low-P conditions. Thus we compared 317 DEGs and 195 marked genes, finding 85 overlapping genes that were considered as active genes induced by low-P stress. Further study revealed most of the common genes focused on roots, which was consistent with a greater number of DEGs in roots than leaves in different $\mathrm{P}$ conditions.

Interestingly, the 10 genes that showed opposite expression patterns in roots all belonged to the overlapping genes (Additional file 5: Table S3). Despite two genes (ProbeSetID:11829195 and 12212657) that could not find corresponding symbol and one gene (ProbeSetID:11858123) that could not find functional annotation, seven genes (GmNAC11 [Glyma16g04740.1], Glyma03g31940.1, Glyma08g20220.1, Glyma13g27820.1, Glyma13g31580.1, Glyma18g53170.1 and Glyma19g32700.1) with functional annotations were shown to be associated with biotic and abiotic stresses.

Likewise, GO and KEGG enrichment analyses were applied to evaluate the potential functions of the 85 overlapping DEGs. DEGs significantly enriched in GO terms of biological processes such as oxidation reduction and response to jasmonic acid stimulus. Molecular functions such as oxidoreductase activity, and cellular components such as the cell wall, were also enriched. Meanwhile, sixteen pathways were enriched based on 0.05 significance level. Similar to the results of the 195 marked genes, the pathways of phenylpropanoid biosynthesis, methane metabolism, phenylalanine metabolism, and flavonoid biosynthesis were also detected (Additional file 2: Table S1).

\section{Significantly differentially expressed genes between roots and leaves}

In our study, under low-P treatment, more DEGs were detected in roots than leaves, suggesting there were more genes in response to low-P stress during seedling stage in roots. DEGs between roots and leaves were found (Table 2). DEGs induced by low-P conditions were classified into three categories according to their expression patterns in normal conditions. For 6370 up- 
Table 2 The number of DEGs in CD/YH and roots/leaves

\begin{tabular}{|c|c|c|c|c|c|}
\hline \multirow[t]{2}{*}{ Expression pattern } & & \multicolumn{2}{|l|}{$\mathrm{CD} / \mathrm{YH}$} & \multicolumn{2}{|c|}{ Roots/leaves } \\
\hline & & Roots & Leaves & $C D$ & $\mathrm{YH}$ \\
\hline Normal $\mathrm{P} \uparrow$ & & 1325 & 3835 & 5945 & 7631 \\
\hline \multirow[t]{4}{*}{ Low $P \uparrow^{a}$} & Normal $\mathrm{P} \uparrow^{\mathrm{b}}$ & 941 & 2636 & 5137 & 6149 \\
\hline & Normal $\mathrm{P} \rightarrow^{\mathrm{c}}$ & $826(93)^{e}$ & $975(40)$ & $1232(97)$ & $1207(63)$ \\
\hline & Normal $\mathrm{P} \downarrow^{\mathrm{d}}$ & 10 & 1 & 1 & 0 \\
\hline & Total & 1777 & 3612 & 6370 & 7356 \\
\hline Normal $P \downarrow$ & & 1091 & 1771 & 5173 & 4799 \\
\hline \multirow[t]{4}{*}{ Low $P \downarrow$} & Normal P $\downarrow$ & 610 & 1040 & 4696 & 4156 \\
\hline & Normal P $\rightarrow$ & $324(14)$ & $526(38)$ & $893(19)$ & $1075(73)$ \\
\hline & Normal $\mathrm{P} \uparrow$ & 0 & 0 & 0 & 0 \\
\hline & Total & 934 & 1566 & 5589 & 5231 \\
\hline
\end{tabular}

a Significantly up-regulated expression pattern under low-P stress;

b Significantly up-regulated expression pattern under normal P condition;

c The expression patterns were not significantly changed under normal P condition;

d Significantly down-regulated expression pattern under normal $P$ condition;

e The number in parentheses indicates the number of genes identified by our criteria (Additional file 4: Figure S2)

regulated genes between roots and leaves in $\mathrm{CD}$ under low-P stress, three categories of DEGs were 5137 upregulated genes, one down-regulated gene, and 1232 genes showing no different expression in roots/leaves in normal conditions. Among the 1232 genes, there were 97 genes that showed larger than 4-fold expression difference between roots and leaves under low-P conditions. Additionally, we found 63,19 , and 73 genes showing larger than 4fold expression differences under low-P conditions from the 1207,893 , and 1075 genes, respectively (Table 2 ). With one opposite-expressed gene, there were 253 marked DEGs between roots and leaves.

We performed the enrichment of GO and KEGG analysis for these 253 marked DEGs. GO analysis showed these genes enriched in a group of biological processes, for example, oxidation reduction, response to oxidative stress, and response to abiotic stimulus. KEGG analysis revealed most of these genes enriched in methane metabolism, phenylpropanoid biosynthesis, and phenylalanine metabolism (Additional file 2: Table S1).

The 253 marked DEGs between roots and leaves were related with low-P stress. There were 317 DEGs in roots and leaves considered to be caused by low-P stress. Thus, we compared 317 DEGs and 253 marked genes and found 68 overlapping genes. Interestingly, 64 overlapping genes were up-regulated in $\mathrm{CD}$ between roots and leaves, consistent with the results that more genes were induced in $\mathrm{CD}$ than $\mathrm{YH}$ under low-P stress. GO enrichment analysis showed the 68 overlapping genes enriched in biological processes such as response to cadmium ion, defense, jasmonic acid stimulus $(P<0.05)$. In the molecular function term and cellular component, these genes were significantly enriched in transcription regulator activity and in the nucleus $(P<0.05)$ (Additional file 2: Table S1). Furthermore, we identified pathways including methane metabolism and flavonoid biosynthesis $(P<0.05)$.

Common DEGs among treatments, materials, and tissues

The 195 marked DEGs between CD and YH under low$P$ stress were identified as DEGs between materials which were associated with low-P stress, while the 253 marked DEGs between roots and leaves in low-P conditions were regarded as DEGs between tissues that were related to low-P stress. We compared the 195, 253 marked DEGs with the 317 DEGs between different $\mathrm{P}$ treatments and 42 common DEGs were found to be in all three classes. That is to say, the 42 DEGs occurred not only between treatments, but also between materials and tissues under low-P stress, which suggested they were active genes under low-P conditions. Further KEGG analyses enriched in three general pathways: methane metabolism, phenylalanine metabolism, and phenylpropanoid biosynthesis. Notably, the 5 genes enriched in the three pathways were all the same. Among them, four genes including Glyma02g40000, Glyma11g29890, Glyma18g06250 and Glyma20g30910 acted as peroxidase to generate $\mathrm{p}$-Hydroxyphenyl lignin ( $\mathrm{H}$ lignin), Guaiacyl lignin (G lignin) and Syringyl lignin (S lignin) from corresponding p-Coumaryl alcohol, Coniferyl alcohol and Sinapyl alcohol (Fig. 2). One gene named Glyma02g14450 functioned in the production of $\mathrm{p}$ Coumaroyl-CoA, which involved in the biosynthesis of flavonoid.

\section{Quantitative Real-time PCR (RT-qPCR) verification}

To verify the expression results obtained by gene chips, a total of 13 differently expressed genes either between 


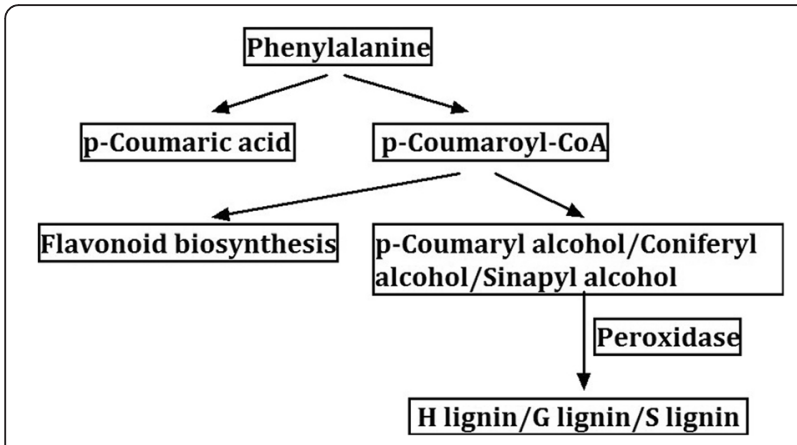

Fig. 2 Common DEGs enriched on phenylpropanoid biosynthesis pathway in soybean

soybean accessions $\mathrm{CD}$ and $\mathrm{YH}$ in low-P stress or between different $\mathrm{P}$ treatments in roots of $\mathrm{CD}$ and $\mathrm{YH}$ were selected for RT-qPCR. The results of RT-qPCR analysis were consistent with the data obtained from gene chips (Fig. 3), indicating the reliability of the results from gene chips.

\section{Discussion}

\section{Low phosphorus induced systemic changes in soybean}

Phosphorus is important to soybean growth and development. To detect low-P-stress related genes, multiple comparative analyses were conducted. First, we compared the expression level changes between different $\mathrm{P}$ treatments in the given materials and tissues, which represented intra-material and intra-tissue DEGs in coping with low-P conditions. Simultaneously, we analyzed inter-materials and inter-tissues gene expression patterns in the case of different concentrations of phosphorus. Finally, we obtained overlapping DEGs between intermaterials and intra-materials, between inter-tissues and intra-tissues, and common genes among different $\mathrm{P}$ treatments, materials and tissues. Through these comparative analyses, we acquired stable genes responding

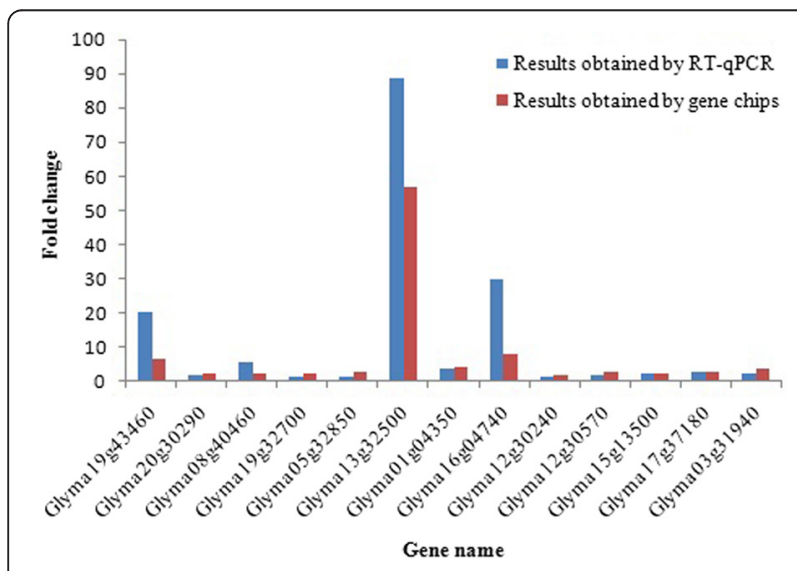

Fig. 3 Validation of the results obtained by gene chips with RT-qPCR to low-P stress, and obtained common genes and pathways related to low-P stress in soybean.

Zeng et al. revealed 17 biological processes in GO enrichment analysis of phosphate-deficiency-responsive genes in soybean roots (Glycine max var. Williams 82) [28]. Among these processes, photosynthesis, iron ion transport, fatty acid metabolic process, and stress responses were also detected in our study. Through the analyses of the GO enrichment results, we found oxidative response, as well as peroxidases were repeatedly detected. As earlier studies demonstrated, the expression levels of peroxidases were closely related to the resistance to biotic and abiotic stresses, such as salt stress, wounding, diseases, hypersensitive reactions, and so on [31-34]. Recent studies confirmed that under P-limited conditions, alterations of oxidative stress- related genes including peroxidase genes were observed in Zea mays, rice and Arabidopsis [23, 24, 27].

Simultaneously, the results of GO enrichment analyses also showed many genes induced by low-P stress were associated with plant hormone signal transduction, such as ethylene and jasmonic acid. Previous studies supported that many genes induced by low-P stress were associated with plant hormone signal transduction [23-25, 35]. An increase of ethylene content in root systems of common bean indicated that ethylene regulated changes in root morphology (i.e. root length) under low-P stress [36]. Meanwhile, the effect of ethylene on the elongation of primary roots in Arabidopsis and the emergence of root hairs under low-P stress was shown with the use of a method based on image processing [37]. Gao et al. studied the effect of ethylene on physiological changes of soybean plants grown in P-deficient solution. Their results suggested that ethylene increased root vigor and acid phosphatase activity in the root system, as well as root:shoot ratio under low-P stress [38]. In addition, jasmonic acid promoted plants' freezing tolerance with the application of exogenous jasmonic acid and blocking-up biosynthesis of jasmonic acid [39].

The three common pathways, methane metabolism, phenylalanine metabolism, and phenylpropanoid biosynthesis, were enriched from comparative analyses, which suggested that these pathways responded actively when plants suffered low-P stress. These results were further supported by the facts that transcript profiling analyses of Zea mays and Arabidopsis identified that many genes involved in biosynthesis of phenylpropanoids either upregulated or down-regulated when exposed to low-P stress $[24,25]$. Phenylpropanoid was a member of phenolic compound, which pertained to plant secondary metabolites. For example, Hall et al. found that the phenolic content was stimulated by an increase in nutrient stress in Helianthus annuns [40]. Similar phenomenon occurred in Tageteserecta when suffering from water stress with a 
significant increase of phenolic content [41]. Koeppe et al. observed that more phenol compounds were leached from living intact roots, dried roots, and tops of phosphatedeficient plants than from phosphate-sufficient ones [42]. Although the effect of methane metabolism on the response to low-P stress had not been clearly stated, a recent study suggested plants released more methane at high temperatures [43], and methane could play an important role in regulating plant growth [44]. Methane metabolism in association with low-P stress may provide a new direction in studying the mechanism of low-P tolerance in soybean.

Our results suggested that the responses of soybean to low-P stress were complex cross-talks; it not only depended on other nutrition (iron) and plant hormones levels (ethylene and jasmonic acid), but also on the metabolism of secondary metabolites. This information can be used to focus on these systemic connections induced by low-P stress to devise strategies aimed at improving soybean yield in P-deficiency soils.

\section{Massive genes involved in response to low-P stress in low-P-tolerant accession}

We selected a low-P-tolerant accession, CD, and a low$\mathrm{P}$-sensitive accession, $\mathrm{YH}$. The numbers of different expression genes between different $\mathrm{P}$ treatments in $\mathrm{CD}$ were 30 -fold more than those in $\mathrm{YH}$. To explore whether the DEGs were accession-specific between $C D$ and $\mathrm{YH}$, we compared the DEGs between these two accessions. Our results showed that among the 10 DEGs in $\mathrm{YH}$, one gene (Glyma20g33710) was also found in CD. A homologous gene was identified in Lotus japonicus (Accession No. AB378629) with the tool of BLAST in the National Center for Biotechnology Information (NCBI) database (http://blast.ncbi.nlm.nih.gov/Blast.cgi), which exhibited $54 \%$ sequence identify to Glyma20g33710. This gene was predicted as a nodulation-associated bZIP transcription factor gene [45]. For a DEG Glyma09g18770 in $\mathrm{YH}$, a homologous gene Glyma17g10510 was detected in the DEGs from CD. Glyma09g18770 encoded a putative E3 ligase protein with metal ion binding and DNA binding domains, homologous with BRUTUS and OsHRZ in Arabidopsis thaliana and Oryza sativa which regulates the response to iron deficiency [46]. The rest annotated DEGs in YH, three genes (Glyma03g28630, Glyma19g31361 and Glyma03g28611) encoded the transcription factor ORG2-like proteins. The homologous gene in Arabidopsis thaliana was bHLHO38, which played an important role in the iron-deficiency responses and uptake of this nutrient [47]. Glyma04g41510 was a plastid-lipid-associated fibrillin protein, transferring phosphorus-containing groups. Glyma20g33710 encoded a member of basic leucine zipper transcription gene family, homologs with TGA4 in Arabidopsis thaliana. TGA4 acts as a regulator in response to defense signals [48]. Although no homologous genes of the rest of the DEGs were detected in $\mathrm{CD}$; gene response to iron-deficiency, uptake, and defense were detected in $\mathrm{CD}$. These genes might be located in different pathways or different locations of the same pathway.

In conclusion, most DEGs were specific between $\mathrm{CD}$ and YH (Additional file 3: Table S2), however, the involved pathways found in $\mathrm{YH}$ were included in $\mathrm{CD}$. In addition, other low-P stress related pathways were detected in $\mathrm{CD}$, for example, oxidation-reduction and metabolic processes. The much more genes induced by low-P stress existed in $\mathrm{CD}$ than in $\mathrm{YH}$ suggested massive genes involved in the response to low-P condition in tolerant accession $\mathrm{CD}$. In addition to involving in biotic and abiotic stress responses, some of these genes were related to plant growth and development. On one hand, these massive genes could response to low-P condition through mediating the secretion of phytohormone and secondary metabolites. On the other hand, they could improve uptake of $\mathrm{P}$ through controlling soybean root traits, such as main root elongation and increased root hair number. All of these responses could make CD more adaptive to low-P stress and more resistant than YH. Likewise, more stress-responsive genes were identified in tolerant soybean line than those in sensitive soybean line in the response to common cutworm feeding [49]. Moreover, more DEGs were detected in CD than those in $\mathrm{YH}$ under low-P stress (Table 1) could be due to different genetic backgrounds between these two materials, which was further supported by the result that there were 64,534 SNPs detected between CD and YH by a genome-wide NJAU $355 \mathrm{~K}$ SoySNP array (unpublished data). The selection of P-efficient soybean accessions could be helpful in exploring low-P related genes and creating new low-P-tolerant soybean accessions.

\section{Roots played a crucial role in $\mathrm{P}$ metabolism during seedling stage}

Compared with 310 DEGs detected in roots under low-P stress, only 18 DEGs were detected in leaves (Table 1 ). Plants under low-P conditions depended on the root system during seedling stage to absorb phosphorus nutrition. Previous studies showed an increase in lateral roots, root hair density, root:shoot ratio when plants were exposed to low-P stress [50, 51]. Among them, uptake through root hairs contributed up to $90 \%$ of the phosphorus acquired by plants [52]. Some phosphate transporters specifically located in roots had been identified to be involved in Pi uptake at the root-soil interface in P-deficient soils. These proteins transport available phosphorus that could be absorbed by plants, mainly $\mathrm{H}_{2} \mathrm{PO}_{4}^{-}, \mathrm{HPO}_{4}^{2-}$ and $\mathrm{PO}_{4}^{3-}$ [53]. The apparent changes of root systems under low-P stress in our hydroponic 
experiments also confirmed that roots played an important role in acquiring P during seedling stage. As such, we found some genes associated with phosphorus absorption in roots. The gene Glyma04g19450 showed homology with AtSPX2-4, members of a sub-family of Arabidopsis genes with the SPX domain. Proteins harboring SPX domain are believed to be involved in phosphorus acquisition and phosphorus signaling network. Duan et al. found several Pi starvation-responsive genes were regulated by AtSPX2-4, positively or negatively. Further studies demonstrated the expression levels of AtSPX2 and AtSPX3 increased under phosphorus starvation [10]. Interestingly, we also found three DEGs in roots; namely Glyma13g24810.1, Glyma07g31630.1 and Glyma13g31290.1 respectively, showed homology with $\mathrm{PHO} 2$, the ubiquitin-conjugating enzyme that contained SPX domain, which is crucial for phosphorus acquisition and translocation in plants. Results of Huang et al. suggested PHO2 modulated Pi absorption, specifically transport at the root surface by regulating the abundance of PHT1s [54].

The limited number of DEGs in leaves probably resulted from genes in the leaves primarily involved in P distribution and utilization in later stages. Three DEGs from leaves (Glyma03g28630, Glyma19g31361 and Glyma03g28611) were homologous to bHLHO38 in Arabidopsis thaliana, functioning in the iron-deficiency responses and uptake [55]. Thus, we concluded roots played an important role in coping with low-P stress during seedling stage. An indepth study on DEGs in roots will advance the functional study of highly P-efficient genes.

\section{Conclusion}

P starvation leads to systemic changes in the gene expression of soybean. Here, the gene expression patterns of soybean under low-P stress were surveyed on the roots and leaves of a low-P-tolerant accession and a lowP-sensitive accession by microarray chips. Through comparative analyses on the differently expressed genes, we identified 42 candidate genes and three common pathways, including methane metabolism, phenylalanine metabolism and phenylpropanoid biosynthesis, which were highly correlated to low-P stress. These results not only promote our understandings of the molecular bases of the responses to $\mathrm{P}$ deficiency, but also facilitate research in improving Pi usage in soybean and designing highly phosphate-efficient soybeans. This process could optimize fertilizer use and promote development of sustainable agricultural practices.

\section{Methods}

\section{Plant materials}

Through hydroponic experiments, we selected two soybean accessions, Chundou (CD) and Yunhefengwodou
(YH), from 219 soybean materials [29]. The 219 soybean seedlings were sown in plastic pots which contained the nutrient soil and vermiculite with a ratio of 1:3. After five days of germination, we picked three shoots of each accession with similar growth vigor which were then transplanted to half Hoagland under different $\mathrm{P}$ treated conditions in hydroponic boxes with a $16 \mathrm{~h} / 8 \mathrm{~h}$ (day/ night) photoperiod and a temperature of $26-28{ }^{\circ} \mathrm{C} / 22{ }^{\circ} \mathrm{C}$ (day/night) temperature cycle. Hoagland nutrient solution was composed of macroelements $\left(1.0 \mathrm{mM} \mathrm{KH}_{2} \mathrm{PO}_{4}\right.$, $\left.5.0 \mathrm{mM} \mathrm{KNO}_{3}, 5.0 \mathrm{mM} \mathrm{Ca}\left(\mathrm{NO}_{3}\right)_{2}, 2.0 \mathrm{mM} \mathrm{MgSO}_{4}\right)$, microelements $\left(2.86 \mathrm{mg} / \mathrm{L} \mathrm{H}_{3} \mathrm{BO}_{3}, 1.81 \mathrm{mg} / \mathrm{L} \mathrm{MnCl}_{2}\right.$. $4 \mathrm{H}_{2} \mathrm{O}, 0.22 \mathrm{mg} / \mathrm{L} \mathrm{ZnSO}_{4} \cdot 7 \mathrm{H}_{2} \mathrm{O}, 0.08 \mathrm{mg} / \mathrm{L} \mathrm{CuSO} 4$. $\left.5 \mathrm{H}_{2} \mathrm{O}, 0.0269 \mathrm{mg} / \mathrm{L} \mathrm{Na} \mathrm{NoO}_{4} \cdot 2 \mathrm{H}_{2} \mathrm{O}\right)$ and ferric salts $\left(5.56 \mathrm{mg} / \mathrm{L} \mathrm{FeSO}_{4} \cdot 7 \mathrm{H}_{2} \mathrm{O}, 7.64 \mathrm{mg} / \mathrm{L}\right.$ EDTA $\left.\cdot \mathrm{Na}\right)$. Hoagland with $1.0 \mathrm{mmol} / \mathrm{L} \mathrm{P}$ was considered as $+\mathrm{P}$ treated condition, while Hoagland with $0.01 \mathrm{mmol} / \mathrm{L} \mathrm{P}$ was considered as -P treated condition. To satisfy the demands of plant growth, we substituted equal concentrations of $\mathrm{KCl}$ for $\mathrm{KH}_{2} \mathrm{PO}_{4}$. Ventilation was performed three times per day for $30 \mathrm{~min}$. Nutrient solution was exchanged every three days.

Then Roots and leaves of soybean accession CD and YH were harvested after 10 days of hydroponics (normal and low $\mathrm{P}$ treated conditions), each with three biological replicates. All samples were stored at $-80{ }^{\circ} \mathrm{C}$ for additional experiments, including RNA isolation and Microarray experiments.

\section{Total RNA isolation and quantitative RT-PCR}

Total RNA was extracted from roots and leaves separately of soybean accessions $\mathrm{CD}$ and $\mathrm{YH}$, each with three biological replicates. A total of 24 samples were isolated to exact RNA according to the manufacturer's instructions with the use of Plant RNA Extract Kit (TianGen, Beijing, China). A total of 50-100 mg leaf and 50-100 mg root were used to isolate RNA for each sample, respectively. Then about $1 \mu \mathrm{g}$ RNA was used to configure $20 \mu \mathrm{L}$ system to synthesize cDNA with the application of HiScript ${ }^{\circ}$ II Q RT SuperMix for qPCR (+gDNA wiper) (Vazyme, Nanjing, China). The constitutive expression gene Gmtublin (GenBank accession number: AY907703) was used as a reference gene for RT-qPCR [56], and each sample was measured with three replicates. The RT-qPCR was conducted on an ABI 7500 real-time PCR system (Applied Biosystems, Forster City, CA, USA) with the use of SYBR Green Realtime Master Mix (Toyobo). The ABI 7500 system Sequence Detection System (SDS) software v.1.4 was applied to analyze the data. The primers used were listed in Additional file 6: Table S4.

\section{Microarray experimental design and data analysis}

To obtain the differently expressed genes under different $\mathrm{P}$ treated conditions in different soybean accessions, we 
used 24 Affymetrix Soybean Gene 1.1 ST Array Strip. These detected 66,473 genes from Glyma1.01 database, each with 19 probes, and 8250 genes from GeneBank database, each with 16 probes. After RNA concentrations quantified by ultraviolet Spectrophotometer (NanoDrop Technologies, ND-1000) and RNA quality assessed by formaldehyde agarose gel electrophoresis, the total mRNA was hybridized on Affymetrix Soybean Gene 1.1 ST array strips. Microarray experiments were performed by CapitalBio Technology. All microarrays were scanned with an Affymetrix scanner named GeneChip ${ }^{\circ}$ Scanner 3000, and images acquired were saved as .JPG pictures. AGCC software (Affymetrix ${ }^{\circ}$ GeneChip ${ }^{\circ}$ Command Console $^{\bullet}$ Software) was applied to convert the image signals to digital signals, which recorded the signal intensity of every probe. After subtracting background and consolidating probe signals, normalization between arrays was carried out using RMA algorithm to remove variances between samples caused by abiologic factors [57]. We compared gene expression quantities between different P-treated conditions and different soybean accessions. Differently expressed genes simultaneously satisfied all of the following criteria: (a) $\mid \log _{2-}$ Ratio $\mid \geq 1$, ratio represented fold change of expression between experimental group control group, (b) the $\mathrm{P}$ value after FDR correction was less than 0.05 , (c) three biological replicates existed. The analyses of differently expressed genes were performed using SAM (significance analysis of microarray). For DEGs between different $\mathrm{P}$ treatments, A1, A2 and A3 were defined as three biological replicates under normal condition $(0.5 \mathrm{mmol} / \mathrm{L} \mathrm{P})$, and A7, A8 and A9 were defined as three biological replicates under low-P condition (0.005 mmol/L P) (Fig. 1).

\section{Gene Ontology (GO) and KEGG pathway enrichment analysis}

Gene Ontology database was a structured typical biological model; it consisted of three terms which were cellular component (CC), biological process (BP) and molecular function (MF). All differently expressed genes were mapped to GO terms in the GO databases (http:// www.geneontology.org/), then calculated GO terms that were significantly enriched in differently expressed genes compared with genome background with the application of hypergeometric distribution. Results showed biological functions that were significantly associated with genes $(P<0.05)$.

The main metabolic pathways and signal transduction pathways that differently expressed genes may be identified by pathway enrichment analysis. KEGG (Kyoto Encyclopedia of Genes and Genomes) database is an important public database associated with pathways which integrateds genomics, biochemistry and system functional omics. Using KEGG PATHWAY as a unit, we found pathways that were significantly associated with differently expressed genes compared with genome background. Expression profile data were analyzed using a Molecule Annotation System (http://mas.capitalbiotech.com/).

\section{Availability of supporting data}

The data sets supporting the results of this article are included within the article and its additional files. The data from the 24 chips are publicly available in the National Center for Biotechnology Information (NCBI) Gene Expression Omnibus (GEO) under accession number GSE78242 (http://www.ncbi.nlm.nih.gov/geo/query/acc. cgi?acc=GSE78242).

\section{Additional files}

Additional file 1: Figure S1. Roots morphological of soybean accession $\mathrm{CD}$ and $\mathrm{YH}$ under low-P condition $(0.005 \mathrm{mmol} / \mathrm{L} \mathrm{P})$ (A) Root morphological before low-P treatments (B) Root morphological after 10 days of low-P treatments. (DOC $190 \mathrm{~kb}$ )

Additional file 2: Table S1. KEGG and Go enrichment analysis of differently expressed genes. (a) 317 DEGs between different $P$ treatments (b) 195 DEGs between different materials (c) 253 DEGs between different tissues (d) 85 overlapping genes between the 195 marked DEGs between $\mathrm{CD}$ and $\mathrm{YH}$ and 317 DEGs in roots and leaves (e) 68 overlapping genes between the 253 marked DEGs between roots and leaves and 317 DEGs in roots and leaves (f) 42 common DEGs. (XLS 86 kb)

Additional file 3: Table S2. The list of DEGs between different $P$ treatments in the roots and leaves of CD and YH. (XLS $55 \mathrm{~kb}$ )

Additional file 4: Figure S2. Identification of differently up-regulated genes in roots between different soybean accessions. (DOC 26 kb)

Additional file 5: Table S3. 10 genes showing opposite expression patterns in roots between different soybean accessions. (DOC $42 \mathrm{~kb}$ )

Additional file 6: Table S4. The list of primers used in this article. (DOC $32 \mathrm{~kb}$ )

\section{Abbreviations}

BP: biological process; CC: cellular component; CD: Chundou; DEGs: Differently expressed genes; GO: Gene Ontology; KEGG: Kyoto Encyclopedia of Genes and Genomes; MF: molecular function; P: phosphorus; QTL: quantitative trait loci; RT-qPCR: Quantitative Real-time PCR;

SDS: Sequence Detection System; YH: Yunhefengwodou.

\section{Competing interests}

The authors declare that they have no competing interests.

\section{Authors' contributions}

DY, HC and DZ conceived and designed the experiments. QW, YY and WD carried out most of the experiments in this paper. QW and JW performed bioinformatics analysis. QW, JW and HC wrote the paper. All authors read and approved the final manuscript.

\section{Acknowledgments}

This work was supported in part by the National Natural Science Foundation of China (31301342, 31370034 and 31301336), Key Transgenic Breeding Program of China (2014ZX08004-003), and Jiangsu Collaborative Innovation Center for Modern Crop Production (JCIC-MCP).

\section{Author details}

${ }^{1}$ National Center for Soybean Improvement, National Key Laboratory of Crop Genetics and Germplasm Enhancement, Nanjing Agricultural University, Nanjing, China. ${ }^{2}$ Collaborative Innovation Center of Henan Grain Crops, College of Agronomy, Henan Agricultural University, Zhengzhou 450002, China. 


\section{Received: 15 October 2015 Accepted: 29 February 2016} Published online: 05 March 2016

\section{References}

1. Raghothama KG. Phosphate acquisition. Annu Rev Plant Physiol Plant Mol Biol. 1999;50:665-93.

2. Schachtman DP, Reid RJ, Ayling SM. Phosphorus uptake by plants: From soil to cell. Plant Physiol. 1998;116(2):447-53.

3. Bureau M, Mederski $H$, Evans $C$. The effect of phosphatic fertilizer material and soil phosphorus level on the yield and phosphorus uptake of soybeans. Agron J. 1953:45:150-4.

4. Howell RW. Phosphorus nutrition of soybeans. Plant Physiol. 1954;29(5):477-83.

5. Cao M, Tong $Z_{\text {, Han }} \mathrm{M}_{t}$ Cheng $\mathrm{H}$. Selection and evaluation of soybean strains with high-efficient utilization phosphorus. Crops. 2001;4:22-4.

6. Holford ICR. Soil phosphorus: Its measurement, and its uptake by plants. Aust J Soil Res. 1997:35(2):227-39.

7. Lopez-Arredondo DL, Leyva-Gonzalez MA, Gonzalez-Morales SI, Lopez-Bucio J, Herrera-Estrella L. Phosphate nutrition: improving low-phosphate tolerance in crops. Anun Rev Plant Biol. 2014;65:95-123.

8. Ticconi CA, Lucero RD, Sakhonwasee S, Adamson AW, Creff A, Nussaume L, Desnos T, Abel S. ER-resident proteins PDR2 and LPR1 mediate the developmental response of root meristems to phosphate availability. Proc Natl Acad Sci U S A. 2009;106(33):14174-9.

9. Rubio V, Linhares F, Solano R, Martin AC, Iglesias J, Leyva A, Paz-Ares J. A conserved MYB transcription factor involved in phosphate starvation signaling both in vascular plants and in unicellular algae. Genes Dev. 2001;15(16):2122-33.

10. Duan K, Yi K, Dang L, Huang H, Wu W, Wu P. Characterization of a sub-family of Arabidopsis genes with the SPX domain reveals their diverse functions in plant tolerance to phosphorus starvation. Plant J. 2008;54(6):965-75.

11. Zhang D, Song H, Cheng H, Hao D, Wang H, Kan G, Jin H, Yu D. The acid phosphatase-encoding gene GmACP1 contributes to soybean tolerance to low-phosphorus stress. PLoS Genet. 2014;10(1):e1004061.

12. Fan C, Wang X, Hu R, Wang Y, Xiao C, Jiang Y, Zhang X, Zheng C, Fu YF. The pattern of Phosphate transporter 1 genes evolutionary divergence in Glycine max L. BMC Plant Biol. 2013:13:48.

13. Song $H$, Yin Z, Chao M, Ning L, Zhang D, Yu D. Functional properties and expression quantitative trait loci for phosphate transporter GMPT1 in soybean. Plant Cell Environ. 2014;37(2):462-72.

14. Bayuelo-Jimenez JS, Gallardo-Valdez M, Perez-Decelis VA, Magdaleno-Armas L, Ochoa I, Lynch JP. Genotypic variation for root traits of maize (Zea mays L.) from the Purhepecha Plateau under contrasting phosphorus availability. Field Crop Res. 2011:121(3):350-62.

15. Bates TR, Lynch JP. Plant growth and phosphorus accumulation of wild type and two root hair mutants of Arabidopsis thaliana (Brassicaceae). Am J Bot. 2000;87(7):958-63.

16. Gahoonia TS, Nielsen NE. Phosphorus (P) uptake and growth of a root hairless barley mutant (bald root barley, brb) and wild type in low- and high-P soils. Plant Cell Environ. 2003;26(10):1759-66.

17. Dai $X$, Wang $Y$, Yang A, Zhang WH. OsMYB2P-1, an R2R3 MYB transcription factor, is involved in the regulation of phosphate-starvation responses and root architecture in rice. Plant Physiol. 2012;159(1):169-83.

18. King KE, Lauter N, Lin SF, Scott MP, Shoemaker RC. Evaluation and QTL mapping of phosphorus concentration in soybean seed. Euphytica. 2013; 189(2):261-9.

19. Liang QA, Cheng XH, Mei MT, Yan XL, Liao H. QTL analysis of root traits as related to phosphorus efficiency in soybean. Ann Bot. 2010;106(1):223-34.

20. Zhang D, Liu C, Cheng H, Kan G, Cui S, Meng Q, et al. Quantitative trait loci associated with soybean tolerance to low phosphorus stress based on flower and pod abscission. Plant Breed. 2010;129(3):243-9.

21. Wu J, Ma F, Lin H, Liu L, Zhong P, Lin W, Dong D, Wang J, Liu D. Assimilate accumulation, photosynthetic characteristics and yield of soybean genotypes with different phosphorus efficiency. Soybean Sci. 2010;29(2):247-50

22. Cheng F, Tu P, Yan X, Wang X, Liao H. Phosphorus nutrition characters for new soybean germplasms with high phosphorus efficiency in acid red soils. Plant Nutrition and Fertilizer Sciences. 2010;16(1):71-81.

23. Misson J, Raghothama KG, Jain A, Jouhet J, Block MA, Bligny R, Ortet P, Creff A, Somerville $\mathrm{S}$, Rolland $\mathrm{N}$, et al. A genome-wide transcriptional analysis using Arabidopsis thaliana Affymetrix gene chips determined plant responses to phosphate deprivation. Proc Natl Acad Sci U S A. 2005; 102(33):11934-9.

24. Calderon-Vazquez C, Ibarra-Laclette E, Caballero-Perez J, Herrera-Estrella L. Transcript profiling of Zea mays roots reveals gene responses to phosphate deficiency at the plant- and species-specific levels. J Exp Bot. 2008;59(9):2479-97.

25. Morcuende R, Bari R, Gibon Y, Zheng W, Pant BD, Blasing O, Usadel B, Czechowski T, Udvardi MK, Stitt M, et al. Genome-wide reprogramming of metabolism and regulatory networks of Arabidopsis in response to phosphorus. Plant Cell Environ. 2007;30(1):85-112.

26. Wasaki J, Yonetani R, Kuroda S, Shinano T, Yazaki J, Fujii F, Shimbo K, Yamamoto K, Sakata K, Sasaki T, et al. Transcriptomic analysis of metabolic changes by phosphorus stress in rice plant roots. Plant Cell Environ. 2003;26(9):1515-23.

27. Li L, Liu C, Lian X. Gene expression profiles in rice roots under low phosphorus stress. Plant Mol Biol. 2010;72(4-5):423-32.

28. Zeng H, Wang G, Zhang Y, Hu X, Pi E, Zhu Y, Wang H, Du L. Genome-wide identification of phosphate-deficiency-responsive genes in soybean roots by high-throughput sequencing. Plant Soil. 2015. doi:10.1007/ s11104-015-2657-4

29. Hu Z, Zhang H, Kan G, Ma D, Zhang D, Shi G, et al. Determination of the genetic architecture of seed size and shape via linkage and association analysis in soybean (Glycine max L. Merr.). Genetica. 2013;141(4-6):247-54.

30. Liu Y, Dammann C, Bhattacharyya MK. The matrix metalloproteinase gene GMMMP2 is activated in response to pathogenic infections in soybean. Plant Physiol. 2001;127(4):1788-97.

31. Lagrimini LM, Rothstein S. Tissue-specificity of tobacco peroxidase isozymes and their induction by wounding and tobacco mosaic-virus infection. Plant Physiol. 1987;84(2):438-42.

32. Miyazawa J, Kawabata T, Ogasawara N. Induction of an acidic isozyme of peroxidase and acquired resistance to wilt disease in response to treatment of tomato roots with 2-furoic acid, 4-hydroxybenzoic hydrazide or salicylic hydrazide. Physiol Mol Plant P. 1998;52(2):115-26.

33. Martinez C, Montillet JL, Bresson E, Agnel JP, Dai GH, Daniel JF, et al. Apoplastic peroxidase generates superoxide anions in cells of cotton cotyledons undergoing the hypersensitive reaction to Xanthomonas campestris pv. malvacearum race 18. Mol Plant Microbe In. 1998;11(11): 1038-47.

34. Eshdat Y, Holland D, Faltin Z, BenHayyim G. Plant glutathione peroxidases. Physiol Plantarum. 1997;100(2):234-40.

35. Wu P. Phosphate starvation triggers distinct alterations of genome expression in Arabidopsis roots and leaves. Plant Physiol. 2003;132(3):1260-71.

36. Borch K, Bouma TJ, Lynch JP, Brown KM. Ethylene: a regulator of root architectural responses to soil phosphorus availability. Plant Cell Environ. 1999;22(4):425-31.

37. Ma Z, Baskin TI, Brown KM, Lynch JP. Regulation of root elongation under phosphorus stress involves changes in ethylene responsiveness. Plant Physiol. 2003;131(3):1381-90.

38. Gao B, Cao C, Li T. Effect of ethylene on the morphology and physiological characteristic of soybean seedlings under phosphate deficiency. Soybean Sci. 2012;31(1):58-63.

39. Hu Y, Jiang L, Wang F, Yu D. Jasmonate regulates the inducer of cbf expression-C-repeat binding factor/DRE binding factor 1 cascade and freezing tolerance in Arabidopsis. Plant Cell. 2013;25(8):2907-24.

40. Hall AB, Blum U, Fites RC. Stress modification of allelopathy of Helianthus annuus $\mathrm{L}$. debris on seedling biomass production of Amaranthus retroflexus L. J Chem Ecol. 1983;9(8):1213-22.

41. Tang CS, Cai WF, Kohl K, Nishimoto RK. Plant stress and allelopathy. In: Inderjit, Dakshini KMM, Einhellig FA, editors. Allelopathy : organisms, processes, and applications. Washington DC: American Chemical Society; 1995. p. 142-57.

42. Koeppe DE, Southwick LM, Bittell JE. The relationship of tissue chlorogenic acid concentrations and leaching of phenolics from sunflowers grown under varying phosphate nutrient conditions. Can J Bot. 1976;54(10):593-9.

43. Bruhn D, Mikkelsen TN, Obro J, Willats WGT, Ambus P. Effects of temperature, ultraviolet radiation and pectin methyl esterase on aerobic methane release from plant material. Plant Biol. 2009;11:43-8.

44. Cui WT, Qi F, Zhang YH, Cao H, Zhang J, Wang R, et al. Methane-rich water induces cucumber adventitious rooting through heme oxygenase1/carbon monoxide and $\mathrm{Ca}^{2+}$ pathways. Plant Cell Rep. 2015;34(3):435-45. 
45. Kouchi H, Shimomura K, Hata S, Hirota A, Wu GJ, Kumagai H, et al. Largescale analysis of gene expression profiles during early stages of root nodule formation in a model legume. Lotus japonicus DNA Res. 2004;11(4):263-74.

46. Selote D, Samira R, Matthiadis A, Gillikin JW, Long TA. Iron-binding E3 ligase mediates iron response in plants by targeting basic Helix-Loop-Helix transcription factors. Plant Physiol. 2015;167(1):273-86.

47. Lopez-Bucio J, Hernandez-Abreu E, Sanchez-Calderon L, Nieto-Jacobo MF, Simpson J, Herrera-Estrella L. Phosphate availability alters architecture and causes changes in hormone sensitivity in the Arabidopsis root system. Plant Physiol. 2002;129(1):244-56.

48. Foley RC, Singh KB. TGA5 acts as a positive and TGA4 acts as a negative regulator of ocs element activity in Arabidopsis roots in response to defence signals. FEBS Lett. 2004;563(1-3):141-5.

49. Wang $Y$, Wang $H$, Fan $R$, Yang Q, Yu D. Transcriptome analysis of soybean lines reveals transcript diversity and genes involved in the response to common cutworm (Spodoptera litura Fabricius) feeding. Plant Cell Environ. 2014;37(9):2086-101.

50. Gahoonia TS, Nielsen NE. Variation in root hairs of barley cultivars doubled soil phosphorus uptake. Euphytica. 1997;98(3):177-82.

51. Nielsen KL, Eshel A, Lynch JP. The effect of phosphorus availability on the carbon economy of contrasting common bean (Phaseolus vulgaris L.) genotypes. J Exp Bot. 2001;52(355):329-39.

52. Bates TR, Lynch JP. The efficiency of Arabidopsis thaliana (Brassicaceae) root hairs in phosphorus acquisition. Am J Bot. 2000:87(7):964-70.

53. Raghothama KG, Karthikeyan AS. Phosphate acquisition. Plant Soil. 2005; 274(1-2):37-49.

54. Huang TK, Han CL, Lin SI, Chen YJ, Tsai YC, Chen YR, et al. Identification of downstream components of ubiquitin-conjugating enzyme PHOSPHATE2 by quantitative membrane proteomics in Arabidopsis roots. Plant Cell. 2013; 25(10):4044-60.

55. Wang N, Cui Y, Liu Y, Fan HJ, Du J, Huang ZA, et al. Requirement and functional redundancy of Ib subgroup bHLH proteins for iron deficiency responses and uptake in Arabidopsis thaliana. Mol Plant. 2013;6(2):503-13.

56. Chen $H$, Seguin P, Archambault A, Constan L, Jabaji S. Gene expression and isoflavone concentrations in soybean sprouts treated with chitosan. Crop Sci. 2009;49(1):224

57. Irizarry RA, Hobbs B, Collin F, Beazer-Barclay YD, Antonellis KJ, Scherf U, et al. Exploration, normalization, and summaries of high density oligonucleotide array probe level data. Biostatistics. 2003;4(2):249-64.

\section{Submit your next manuscript to BioMed Central and we will help you at every step:}

- We accept pre-submission inquiries

- Our selector tool helps you to find the most relevant journal

- We provide round the clock customer support

- Convenient online submission

- Thorough peer review

- Inclusion in PubMed and all major indexing services

- Maximum visibility for your research

Submit your manuscript at www.biomedcentral.com/submit 